\title{
A Study on OSS Marketing and Communication Strategies
}

\author{
Vieri del Bianco, Luigi Lavazza, Valentina Lenarduzzi, \\ Sandro Morasca, and Davide Taibi, and Davide Tosi \\ Universita' degli Studi dell'Insubria, \\ Dipartimento di Scienze Teoriche e Applicate (DISTA) \\ via Mazzini, 5 Varese, Italy \\ \{vieri.delbianco, luigi.lavazza, valentina.lenarduzzi, \\ sandro.morasca, davide.taibi, davide.tosi\}@uninsubria.it
}

\begin{abstract}
The goal of every open source project is to gain as many satisfied users as possible. To this end, open source software producers should focus on both product development and communication. Currently, most open source projects are mainly concerned with developing code using the most appealing technologies and introducing fancy features. On the contrary, open source software producers seem to lack good communication strategies. In this paper we describe the communication strategies adopted by three successful companies that are active in open source software development. The goal of the paper is to provide some hints that could help other open source software producers identify communication strategies that are effective in promoting their products on the market.
\end{abstract}

\section{$1 \quad$ Introduction}

The usage of Open Source Software (OSS) has been continuously increasing in the last few years, mostly because of the success of a number of well-known projects. However, the diffusion of OSS products is still limited if compared to Closed Source Software (CSS) products. Most consumers are still hesitant in the adoption of OSS. Final users are often skeptical in trusting and adopting software products that are typically developed for free by communities of volunteer developers, who are not supported by large business companies. Moreover, OSS developers often do not pay attention to communication, marketing, commercial, and advertising aspects, since these activities require a huge amount of effort without being gratifying. For most developers, creating attractive websites and leaflets, or spending time on social advertisement is not as much fun as coding might be.

In this work, we analyze marketing and communication strategies of three OSS producers, namely a large software company, a SME and an Open Source Community. Our goal is to provide OSS producers and maintainers with a set of case studies concerning effective and low-effort marketing strategies on how to promote and advertise their OSS products. 
The remainder of this paper is structured as follows. In Section 2, we report a concise literature review. Section 3 reports on the case studies analyzed. Finally in Section 4 we draw conclusions and outline future work.

\section{$2 \quad$ Literature Review}

Although there is a good deal of research on marketing in general, when it comes to software, traditional marketing and communication strategies still mainly focus on CSS [1]. Since implementing a complete marketing strategy is quite a complex process, large industries have undeniable advantages over smaller ones.

SMEs, often managed by technical people, usually adopt simplified marketing strategies because of lack of resources, skills [2], and marketing expertise [4]. Thus, marketing strategies are in some cases based on managers' decisions or personal preference, instead of a strategic and objective assessment of the environment [5].

Moreover, software products evolve quickly, often with decreasing intervals between releases, and become obsolete in a short time [3]: this fast and competitive market pushes companies to focus on product development rather than marketing aspects.

Usually, people who would like to adopt an OSS product are influenced both by opinions and the website of the product itself [6]. The website acts like a shopping window: so, if it is not effective in adequately and completely displaying information on the OSS product, it has very low probability of attracting customers [6]. In fact, the quality of a website is very important for the success of an OSS product, as websites are the main information centers for OSS products.

Moreover, as in CSS, OSS often relies on viral marketing. In viral marketing users are personally involved in the process of acquiring new customers as they are influenced by a sort of "love" for the product and act as direct promoters of the product itself. In this case, users directly encourage friends, relatives and acquaintances to acquire or adopt a product and refer them to the project website from which the OSS product can be downloaded [7]. This strategy is largely adopted by several OSS projects such as Debian, Postgres and Drupal: users act as promoters of the product, for instance by spontaneously adding a footer message to emails advertising the product they are in love with [7].

\section{Case Studies}

In this section, we analyze the marketing strategies adopted by two companies and an OSS community when they launch a new product on the market. We carried out several semi-structured interviews with people, ranging from CEOs to marketing manager about marketing strategies applied to both OSS and CSS. During the interviews we asked the following questions:

Q1: Number of company employees

Q2: How do you come up with ideas for new products or services? 
Q3: On what grounds do you decide to develop a product or not? Do you decide by studying the results of a market analysis or do you make the decision yourself?

Q4: How do you decide if a new project should be released with an OSS license?

Q5: Which marketing strategies do you take into account during the lifecycle of a product or a service?

Q6: Which communication channels do you adopt?

All interviewees also expressed their own opinion on OSS in general and on which strategy should be applied in order to succeed on the market with OSS.

The software company we analyzed is Engineering S.p.A. (ENG). ENG is quoted at the Milan Stock Exchange and generated a revenue above $750 \mathrm{M} €$ in 2010. Engineering is a global player and Italy's largest systems integration group and a leader in the provision of complete integrated services throughout the software value chain. We first interviewed the director of Communication and Marketing to get general information on the adoption of strategies concerning CSS products and services for the whole company. Then, we interviewed the Architectures \& Consulting Director of the Research \& Innovation Division of the ENG group in Padua (Italy) and founder of SpagoWorld (www.spagoworld.org), the free/open source initiative by ENG. SpagoWorld includes six OSS projects: SpagoBI, Spagic, Spago4Q and Spago, available in the OW2 Consortium forge, and eBAM, eBPM available in the Eclipse Foundation forge. The initiative adopts a 100\% open source version (LPGL).

The SME case study was carried out by interviewing professionals of QWERTY S.r.L., an Italian SME located in Pavia. QWERTY has grown its revenue over the last years with an average annual rate of $6.6 \%$ up to $1.2 \mathrm{M} €$ in 2010 . QWERTY has a team of professional a remarkable experience in different platforms, both CSS (Microsoft, IBM, Oracle and others) and OSS (Linux). Some of QWERTY employees have worked for many years in the development of software applications, including integrated ERP systems such as Adempiere, which is also funding partner of the Italian Community (Adempiere Italia). QWERTY does not have employees who are competent in commercial communication, so it outsources marketing and communication to an external communication agency.

We also took analyzed marketing and communication strategies of Ubuntu -one of the biggest OSS communities- by interviewing a member of the Italian Ubuntu marketing and promotion group. Ubuntu is led by Canonical Ltd, which estimates that the product has over 12 million users worldwide and is used to run more than ten thousand applications. Moreover, Canonical supports the user community with websites providing tutorials, a paid support option, and much more. Canonical delivers two Ubuntu releases per year, co-ordinates security and trouble-shooting, and provides an online platform for community interaction. In this work, we took into account the entire work carried out both by Canonical and the community.

Table 1 schematically shows the results of the interviews. 
Table 1. Case Study comparison

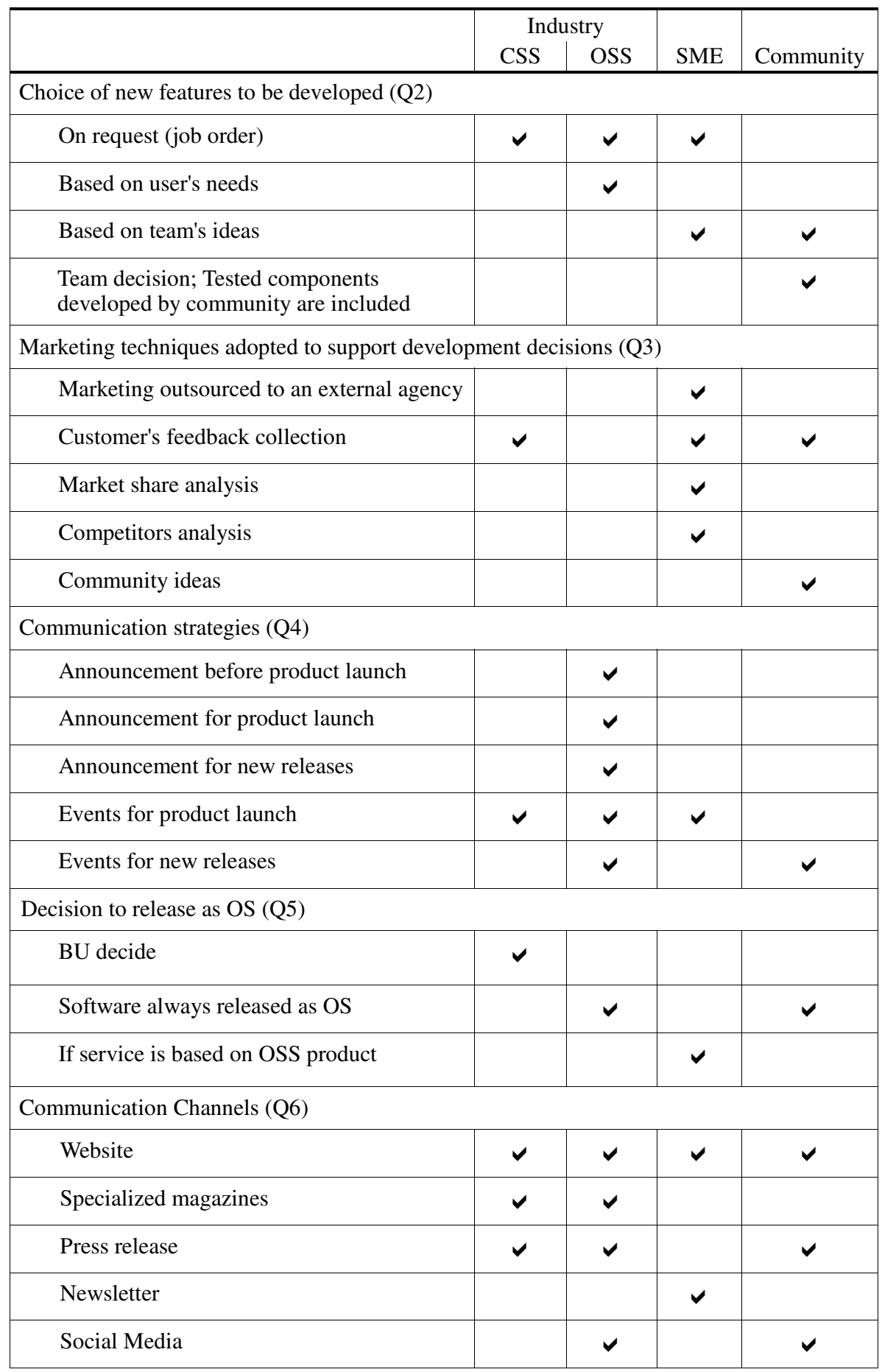


The result showed different approaches to communication. Looking at how different organizations come up with ideas for new products or services, industry and SME introduce new features only in case of job orders, while Ubuntu follows decisions by Canonical, and integrates community developed components only if they are already tested.

Ubuntu community and ENG OSS BU develop only OSS, while for QWERTY the decision to release software as OSS depends on the service requested.

The role of marketing before product release is marginal for the ENG CSS BU, while the OSS BU does not apply marketing strategies at all. Marketing is fundamental for QWERTY, which employs a communication agency for analyzing the market and making surveys on potential competitors. For Ubuntu, marketing is performed by Canonical, and ideas from the community are rarely taken into account.

During the lifecycle of a product or service, ENG CSS BU makes a survey only on already published services and products. On the contrary ENG OSS BU, QWERTY and Ubuntu issue an announcement two months before the product launch and plan some events.

Websites and specific events are adopted by all the interviewed organizations for communication and are considered very useful. Ubuntu is oriented also to using online channels, like twitter and forums, while QWERTY takes advantage of newsletters as well. Both the OSS and CSS business units of ENG exploit the traditional communication channel when the client is a Public Administration and the online channels otherwise.

\section{Conclusion}

This is a unique study comparing OSS communication models which illustrate communication strategies employed by different OSS producers.

The results shown different approaches used in the case studies with some similarities in SME and big industry on communication channel and several differences from the open community.

Of course, this work leaves room for further research. In fact additional case studies, including failure cases, could be explored in the future to further confirm or complement our findings.

Finally, correlations between communication, project diffusion and project maturity could be investigated further.

Acknowledgements. The research presented in this paper has been partially funded by the FIRB project "ARTDECO," sponsored by the Italian Ministry of Education and University and by the project "Metodi e tecniche per l'analisi, l'implementazione e la valutazione di sistemi software" funded by the Università degli Studi dell'Insubria. We are especially grateful to Costanza Amodeo and Gabriele Ruffatti of Engineering group, Adelio Trabella of QWERTY, Monia Spinelli and Paolo Sammicheli of the Ubuntu Foundation. 


\section{References}

[1] Helander, N., Ulkuniemi, P.: Marketing challenges in the software component business. International Journal of Technology Marketing 1(4), 375-392 (2006)

[2] Dwyer, M., Gilmore, A., Carson, D.: Innovative marketing in SMEs. European Journal of Marketing 43(1/2), 46-61 (2009)

[3] Kulmala, H.I., Uusi-Rauva, E.: Network as a business environment: experiences from the software industry. Supply Chain Management: An International Journal 10(3), 169-178 (2005)

[4] Simpson, M., Padmore, J., Taylor, N., Frecknall-Hughes, J.: Marketing in small and medium sized enterprises. International Journal of Behaviour \& Research 12(6), 361-387 (2006)

[5] Chaston, I.: Small firm performance: assessing the interaction between entrepreneurial style and organizational structure. European Journal of Marketing 31(11/12), 814-831 (1997)

[6] Lavazza, L., Morasca, S., Taibi, D., Tosi, D.: OP2A: How to Improve the Quality of the Web Portal of Open Source Software Products. In: Filipe, J., Cordeiro, J. (eds.) WEBIST 2011. LNBIP, vol. 101, pp. 149-162. Springer, Heidelberg (2012)

[7] Guo, S., Wang, M., Leskovec, J.: The role of social networks in online shopping: information passing, price of trust, and consumer choice. In: ACM Conference on Electronic Commerce 2011, pp. 157-166 (2011) 